\title{
BMJ Open Individual and neighbourhood socioeconomic inequalities in cognitive impairment: cross-sectional findings from the French CONSTANCES cohort
}

\author{
Noémie Letellier (D) , Isabelle Carrière, ${ }^{1}$ Emmanuelle Cadot, ${ }^{2}$ Lisa Berkman, ${ }^{3}$ \\ Marcel Goldberg, ${ }^{4,5}$ Marie Zins, ${ }^{4,5,6}$ Claudine Berr ${ }^{1,7}$
}

To cite: Letellier N, Carrière I, Cadot $\mathrm{E}$, et al. Individual and neighbourhood socioeconomic inequalities in cognitive impairment: cross-sectional findings from the French CONSTANCES cohort. BMJ Open 2020;10:e033751. doi:10.1136/ bmjopen-2019-033751

- Prepublication history and additional material for this paper are available online. To view these files, please visit the journal online (http://dx.doi. org/10.1136/bmjopen-2019033751).

Received 26 August 2019 Revised 05 February 2020 Accepted 04 March 2020
Check for updates

(C) Author(s) (or their employer(s)) 2020. Re-use permitted under CC BY-NC. No commercial re-use. See rights and permissions. Published by BMJ.

For numbered affiliations see end of article.

Correspondence to Dr Noémie Letellier; noemie.letellier@inserm.fr

\section{ABSTRACT}

Objectives Despite the recent awareness of the environment impact on brain ageing, the influence of the neighbourhood socioeconomic status on cognitive impairment remains unclear. Here, we investigated the effects of individual and neighbourhood deprivation on cognitive impairment in middle-aged and young-old people. Design Cross-sectional study.

Settings 21 Health Screening Centres in the entire French metropolitan territory.

Participants A total of 44648 participants (age range: 45 to 69 years) from the French CONSTANCES cohort were included in the analyses.

Main outcomes Associations between the overall cognitive score (based on a standardised battery of cognitive tests administered by neuropsychologists) and individual deprivation (Evaluation of Deprivation and Inequalities in Health Screening Centres; EPICES score) and geographical deprivation (French Deprivation Index; FDep index).

Results Based on the EPICES score (validated cutoff $\geq 30.17$ ), $12 \%$ of participants were considered to be deprived. After mutual adjustment, individual and geographical deprivation were associated with higher cognitive impairment in a multilevel logistic regression analysis that was also adjusted for sociodemographic, lifestyle and health factors. Specifically, individual deprivation was associated with an odds increase of $55 \%(\mathrm{OR}=1.55,95 \% \mathrm{Cl}: 1.45$ to 1.66$)$. The risk of global cognitive impairment progressively increased with the neighbourhood deprivation level, evaluated by the FDep index (reference Q1; Q2: $\mathrm{OR}=1.09,95 \% \mathrm{Cl}$ : 0.98 to 1.20 ; Q3: $\mathrm{OR}=1.15,95 \% \mathrm{Cl}: 1.04$ to $1.27 ; \mathrm{Q} 4: \mathrm{OR}=1.15,95 \% \mathrm{Cl}$ : 1.04 to $1.28 ; \mathrm{Q} 5: \mathrm{OR}=1.25,95 \% \mathrm{Cl}: 1.13$ to 1.39 ).

Conclusion Our results suggest that the neighbourhood socioeconomic deprivation level is associated with cognitive impairment, independently of the individual deprivation level. A better understanding of this association could help to define new prevention strategies to target high-risk residents and high-risk geographical areas in order to reduce social health inequalities.

\section{INTRODUCTION}

The concept of deprivation was defined by Townsend as a 'state of observable and
Strengths and limitations of this study

- We focused on a large population of middle-aged and young-old individuals (age range: 45 to 69 years).

- A cognitive test battery was administered by neuropsychologists in standardised conditions.

- We estimated a gradient of cognitive impairment associated with both individual and neighbourhood deprivation levels.

- Due to the study observational design, we could perform only a cross-sectional analysis of the cognitive performance data.

demonstrable disadvantage relative to the local community or the wider society to which an individual, family, or group belongs'. ${ }^{1}$ Deprivation reflects the accumulation of social and material disadvantages, and is the main cause of health inequalities. ${ }^{23}$ Due to the rapid increase of the proportion of older adults, health inequalities in this population have become a public health priority; however, studies on this topic are still rare. ${ }^{4}$

At the individual level, low socioeconomic status is associated with poorer health, ${ }^{5}$ excess death rate, ${ }^{6}$ increased risk of dementia and lower cognitive performance, independently of the health conditions. ${ }^{78}$ Social health inequalities can also be determined by the deprivation of the residential environment. Indeed, low geographical socioeconomic status has been related to risky health behaviours, cardiovascular disorders and higher mortality. ${ }^{9}$

Recent studies have reported associations between area-level socioeconomic status and cognitive impairment. However, findings on the relationship between neighbourhood deprivation and cognition remain controversial, often due to differences in study design and statistical methods (eg, some studies took 
into account the competing risk of death, while others did not) ${ }^{10}$ One study reported no association of neighbourhood deprivation with cognitive decline in the elderly, ${ }^{11}$ and a recent work with a long follow-up found no association with the risk of dementia. ${ }^{12}$ Conversely, other studies showed that the area-level socioeconomic status might influence cognition, independently of individual characteristics. A deprived residential environment could increase the risk of poor cognitive performance, ${ }^{13}$ cognitive decline ${ }^{1415}$ and dementia, ${ }^{16-18}$ mostly in older $(>65$ years) subjects. This suggests that the residential environment characteristics might be a source of differences in cognitive function in older adults, but probably also earlier in life. Indeed, environmental stimuli influence ageing, but also shape the brain development and affect mental health. ${ }^{19}$ The area where one person lives is characterised by the density and choice of local resources and services (ie, social and physical environment), and can act as a source of complex cognitive stimulation. However, little attention has been given to the effects of the residential environment stimuli on cognitive impairment. ${ }^{20}$

This study investigated the influence of the individual and neighbourhood deprivation levels on cognitive impairment in a large sample of people aged 45-69years living in France. Our main objective was to determine whether the neighbourhood deprivation level was still associated with cognition after taking into account the individual deprivation level and other confounding factors.

\section{METHODS}

\section{Population}

Data were from the French CONSTANCES cohort, a large population-based prospective cohort of 200000 adults aged 18 to 69 years at inclusion. Details on cohort recruitment and data collection are available elsewhere. ${ }^{21}$ Participants were randomly selected among people covered by the Caisse Nationale d'Assurance Maladie health insurance that includes $85 \%$ of the French population (salaried workers, professionally active or retired, and their family). At baseline, health examinations were performed in 21 health screening centres (HSC) in the entire French metropolitan territory. Socioeconomic, demographic, life events and lifestyle data were collected at inclusion using a self-administered questionnaire.

For this study, only those participants who were $\geq 45$ years old and underwent a standardised cognitive assessment performed by trained neuropsychologists were selected. Our analyses were carried out using the data available at the end of January 2019.

\section{Patient and public involvement statement}

There was no patient and public involvement.

\section{Individual deprivation}

The individual socioeconomic status was evaluated using the deprivation score Evaluation of Deprivation and
Inequalities in Health Screening Centres 'EPICES'. ${ }^{22}$ This score was obtained using a validated multidimensional self-questionnaire developed by French HSC experts. It takes into account the multifactorial dimensions of deprivation (material and social conditions), ${ }^{23}$ and is strongly correlated with the Townsend and Carstairs index. ${ }^{6}$ To calculate this score, 11 binary questions about marital status, financial security, access to leisure or sport activities and social support were associated with a weight determined by principal component analysis (PCA) (online supplementary table 1). Given the large number of missing data for the question 'Do you have an additional insurance?' (56\% of non-response), an imputation was performed with the monotone logistic regression method. The EPICES score ranges from 0 (no deprivation) to 100 (maximum deprivation), with a validated deprivation cut-off of $30.17^{22}$ (deprived $\geq 30.17 /$ not deprived <30.17).

\section{Neighbourhood deprivation}

Postal addresses were geocoded to match participants to their residential commune (municipality). The neighbourhood socioeconomic deprivation level was evaluated with a composite French Deprivation Index (FDep index) provided by the French National Institute of Statistics and Economic Studies (INSEE). ${ }^{24}$ This index was built at the commune level using data from the 2009 French census. The FDep index was defined as the first component of the PCA of the following four variables: median household income, percentage of high school graduates in the $\geq 15$-year-old population, percentage of blue-collar workers in the active population, and unemployment rate. This index was calculated for each commune of the CONSTANCES cohort $(\mathrm{n}=4996)$, and was categorised in quintiles. Q1 was considered the reference (the least deprived commune) and Q5 the most deprived residential environment.

\section{Cognitive function}

Cognitive function was assessed at baseline by trained neuropsychologists using a battery of cognitive tests as previously detailed ${ }^{25}$ : the Mini Mental State Examination (MMSE) to assess the global cognitive functioning; the Free and Cued Selective Reminding Test (FCRST) to estimate episodic verbal memory; the Verbal Fluency Tasks (VFT) to evaluate language abilities (semantic and phonemic fluency tasks); the Trail Making Test (TMT-A and TMT-B) coded as time in seconds, to assess executive functions and shifting abilities; and the Digit Symbol Substitution Test (DSST), a subtest of the Wechsler Adult Intelligence Scale-Revised, to explore attention, psychomotor speed and reasoning.

Each specific cognitive skill was analysed separately. For all tests, cognitive impairment was defined by a score below or equal to the 25th percentile of the distribution (higher than or equal to the 75th percentile for TMT) using previously established norms, according to age, sex and education. ${ }^{25}$ 
PCA was used to define a global cognitive score that took into account all cognitive domains. This score was defined as the first axis of the PCA (using the Kaiser criterion) of the scores of the six cognitive tests: FCRST, VFT (semantic and phonemic), TMT-A and TMT-B, and DSST. The first axis explained $47 \%$ of the variance and was characterised by positive scores and high weights for FCRST, VFT (semantic and phonemic) and DSST, and by negative scores for TMT-A and TMT-B. The participants' position on this axis defined their degree of cognitive performance: lower scores indicated worse cognitive performance. Individuals with a score equal or below the adjusted 25th percentile of the sample distribution were classified as 'impaired'.

\section{Covariates}

The following individual covariates were included in the analysis: sociodemographic factors (sex, age (in quintiles: $45-50 />50-55 />55-<60 / 60-<65 / \geq 65)$, geographical origin (proxy of ethnicity, in accordance to French laws), education level ( $<5$ years, 5 to 11 years, 11 to 13 years, 14 to 16 years and 17 years or more) and HSC), lifestyle factors (smoking (never/past/current), alcohol consumption assessed with the AUDIT questionnaire (abstinent/ neither abuse or dependence/abuse/dependence) and body mass index (BMI, $\mathrm{kg} / \mathrm{m}^{2}$; underweight: $\mathrm{BMI}<18.5$, normal: $18.5 \leq \mathrm{BMI}<25$, overweight: $25 \leq \mathrm{BMI}<30$, obesity: BMI $\geq 30$ )), health factors (high blood pressure (HBP; blood pressure higher than $140 / 90 \mathrm{~mm} \mathrm{Hg}$ or history of hypertension), history of diabetes, cardiovascular diseases (CVD; stroke, angina pectoris, myocardial infarction, arteritis of the lower limbs, others) and depressive symptomatology defined as a Center for Epidemiological Studies-Depression (CES-D) scale score $\geq 16$ ).

In additional analyses, the rural/urban status of the commune was considered, according to the classification by the INSEE. Individual variables were included in supplementary analyses: self-reported daily mobility (limited daily mobility was defined when the participant reported problems in using transport, going up or down a flight of stairs, or walking $1 \mathrm{~km}$ without stopping), physical activity (score from 0 to 6; 0 for inactive people and six for very active people) and social isolation (contact with family or friends $\leq 2$ times per month).

\section{Statistical analyses}

Considering the data hierarchical structure, a multilevel logistic regression model was used to take into account the clustering of individuals through the introduction of a cluster-specific random effect for the commune. ${ }^{26}$ Laplace's method was used to estimate parameters. This method includes an approximation of the likelihood and is prone to effect underestimation.

For each cognitive test, including the global cognitive score, dichotomised at the 25 th percentile (75th percentile for TMT), a multilevel logistic regression model was used to estimate the OR and the $95 \%$ CI. First, individual deprivation (model 1) and neighbourhood deprivation (model 2) were modelled separately. Sex, age, education level, HSC, geographical origin, smoking, alcohol consumption, BMI, HBP, diabetes, CVD and CES-D score were introduced in model 1 and model 2 . The last model (model 3) considered together the individual deprivation and neighbourhood deprivation scores adjusted for individual characteristics. Model 3a included major confounders of cognition: sex, age and education level. Model 3b included the HSC and other confounders (geographical origin, smoking, alcohol consumption, BMI, HBP, diabetes, CVD and CES-D). All analyses were performed using SAS V.9.4.

\section{RESULTS}

\section{Study population}

Analyses concerned only French-speaking participants aged 45-70years with available cognitive data at the end of January 2019, and no missing data for the EPICES score and covariates (see sample selection in online supplementary figure 1). Among the 44648 participants included in the sample, $52 \%$ were women and the median (IQR) age at enrolment was 57.5 years (51.5-63.5). Compared to the excluded people, included participants were more often men $(\mathrm{p}=0.001)$, younger, more educated and wealthier $(\mathrm{p}<0.0001)$.

According to the EPICES score, $12.5 \%$ of participants were personally deprived $(n=5565)$. Deprived individuals were younger ( 56.5 vs 57.5 years, $\mathrm{p}<0.0001$ ) (table 1 ), whereas the proportion of women was similar in the deprived and not deprived groups $(\mathrm{p}=0.27)$. Overweight, history of CVD, HBP and depressive symptoms were more frequent in the deprived group $(\mathrm{p}<0.0001)$. Cognitive performance scores (all tests) were lower in the deprived group (table 1). Moreover, individually deprived people lived more often in a deprived neighbourhood compared with people who were not individually deprived $(25.4 \%$ vs $19.2 \%$ lived in the most deprived neighbourhood).

In comparison with people living in the least deprived area $(\mathrm{Q} 1 ; \mathrm{n}=8902)$, people living in the most deprived environment (Q5; $\mathrm{n}=8929$ ) were more frequently men (50\% vs $46 \%, \mathrm{p}<0.0001)$ and had a lower education level (14\% had less than 11 years of education vs $6 \%$, $\mathrm{p}<0.0001)$. They tended to have higher BMI $(17 \%$ were obese vs $10 \%, \mathrm{p}<0.0001)$, and history of hypertension (45\% vs $35 \%, p<0.0001)$, CVD and diabetes $(\mathrm{p}<0.0001)$.

Cognitive performances were lower in people living in the most deprived area compared with those who lived in the least deprived area, as indicated by the lower median score of all cognitive tests: MMSE (28 vs 29), FCRST (32 vs 34), semantic VFT (23 vs 24), phonemic VFT (14 vs 16), DSST score (65 vs 69) and for TMT-A (32 vs 31) and TMT-B (61 vs 57). Moreover, cognitive performance (global cognitive score) decreased in function of the neighbourhood deprivation level (figure 1) in the individually deprived and also in the non-individually deprived group. 
Table 1 Population characteristics according to the individual deprivation $(n=44648)$

Individual characteristics, $\mathbf{n}(\%)$

Sociodemographic and socio-economic factors

Women

Age (years) $\dagger$

Years of education

$>5$

5 to 11

11 to 13

14 to 16

$\geq 17$

Lifestyle and health factors

Alcohol

Abstinent

No abuse or dependence

Abuse

Dependence

Smoking status

Never

Current

Past

BMI $\left(\mathrm{kg} / \mathrm{m}^{2}\right)$

Underweight $(<18.5)$

Normal $(18.5-<25)$

Overweight $(\geq 25-30)$

Obese (>30)

Hypertension

Diabetes

History of cardiovascular disease

Depressive syndrome

Cognitive performance $\dagger$

MMSE (0-30)‡

FCRST (Total free recall 0-48)‡

Semantic VFT (words in $1 \mathrm{~min}$ )‡

Phonemic VFT (words in $1 \mathrm{~min}) \ddagger$

TMT A (max 180s)‡

TMT B $(\max 180 \mathrm{~s}) \ddagger$

DSST score (0-135)‡

Neighbourhood characteristics, $n(\%)$

FDep deprivation index

$\begin{array}{ll}\text { Q1 (least deprived) } & 8000(20.5) \\ \text { Q2 } & 7978(20.4) \\ \text { Q3 } & 7947(20.3) \\ \text { Q4 } & 7644(19.6) \\ \text { Q5 (most deprived) } & 7514(19.2)\end{array}$

Not deprived $(n=39083)$

Deprived $(n=5565)$

P value*

$\begin{array}{cc}20217(51.7) & 2923(52.5) \\ 57.5(51.5-63.5) & 56.5(50.5-6 \\ & \\ 634(1.6) & 368(6.6) \\ 2715(7.0) & 750(13.5) \\ 14592(37.3) & 2832(50.9) \\ 13341(34.1) & 1196(21.5) \\ 7801(20.0) & 419(7.5)\end{array}$

$<0.0001$

$<0.0001$

$\begin{array}{cc}841(2.2) & 318(5.7) \\ 31776(81.3) & 4087(73.4) \\ 5312(13.6) & 772(13.9) \\ 1154(3.0) & 388(7.0)\end{array}$

$17720(45.3)$

0.02

$4532(11.6)$

2125 (38.2)

$1302(23.4)$

$2138(38.4)$

$16831(43.1)$

$1050(2.7)$

$163(2.9)$

$19216(49.2)$

$2135(38.4)$

$14002(35.8)$

$2012(36.2)$

$4815(12.3)$

$1255(22.6)$

$15281(39.1)$

$2583(46.4)$

$<0.0001$

987 (2.5)

317 (5.7)

$<0.0001$

3489 (8.9)

609 (10.9)

$<0.0001$

6302 (16.1)

2245 (40.3)

$<0.0001$

$\begin{array}{lll}29(28-30) & 28(27-29) & <0.0001 \\ 33(29-37) & 32(28-36) & <0.0001 \\ 24(20-28) & 22(18-26) & <0.0001 \\ 15(12-19) & 14(11-17) & <0.0001 \\ 31(25-39) & 33(27-42) & <0.0001 \\ 59(47-74) & 66(52-87) & <0.0001 \\ 68(58-77) & 62(52-72) & <0.0001\end{array}$

$<0.0001$

902 (16.2)

1000 (18.0)

992 (17.8)

1256 (22.6)

1415 (25.4) 
Table 1 Continued

\begin{tabular}{llll}
\hline Individual characteristics, $n(\%)$ & Not deprived $(n=39083)$ & Deprived $(n=5565)$ & $P$ value*
\end{tabular}

${ }^{*} P$ value adjusted for sex and age.

†Median (IQR).

$\ddagger$ Theoretical range.

BMI, body mass index; DSST, Digit Symbol Substitution Test; MMSE, Mini Mental State Examination.

\section{Individual and neighbourhood deprivation: associations with cognitive impairment}

In multivariate analyses (model 1 adjusted for sex, age, ethnicity, education, HSC, health behaviours and health status), the risk of global cognitive impairment was higher for deprived individuals (EPICES score $\geq 30.17$ ) (OR, $1.56 ; 95 \%$ CI 1.46 to 1.67 ) (table 2 ).

Similarly, the risk of cognitive impairment was higher for people living in a deprived environment (model 2 adjusted for sex, age, ethnicity, education, HSC, behaviours and health status) (table 2). This risk was higher for people living in areas with higher levels of neighbourhood deprivation (ref. Q1; Q2: OR=1.09, $95 \%$ CI: 0.98 to 1.20 ; Q3: OR $=1.15,95 \%$ CI: 1.04 to 1.27 ; Q4: $\mathrm{OR}=1.17,95 \%$ CI: 1.05 to 1.29 ; $\mathrm{Q} 5: \mathrm{OR}=1.28,95 \% \mathrm{CI}$ : 1.15 to 1.41$)$.

No interaction was observed between neighbourhood deprivation and individual characteristics (sex, age, ethnicity, education level, daily mobility, social isolation and individual deprivation).

When the two deprivation scores were considered in the same model, they remained highly significant (model 3; figure 2). Model 3 highlighted the influence of neighbourhood deprivation on cognition, independently of the individual deprivation score. After adjustment for confounders and individual deprivation (model 3b), the risk of cognitive impairment remained higher for people living in areas with higher levels of neighbourhood

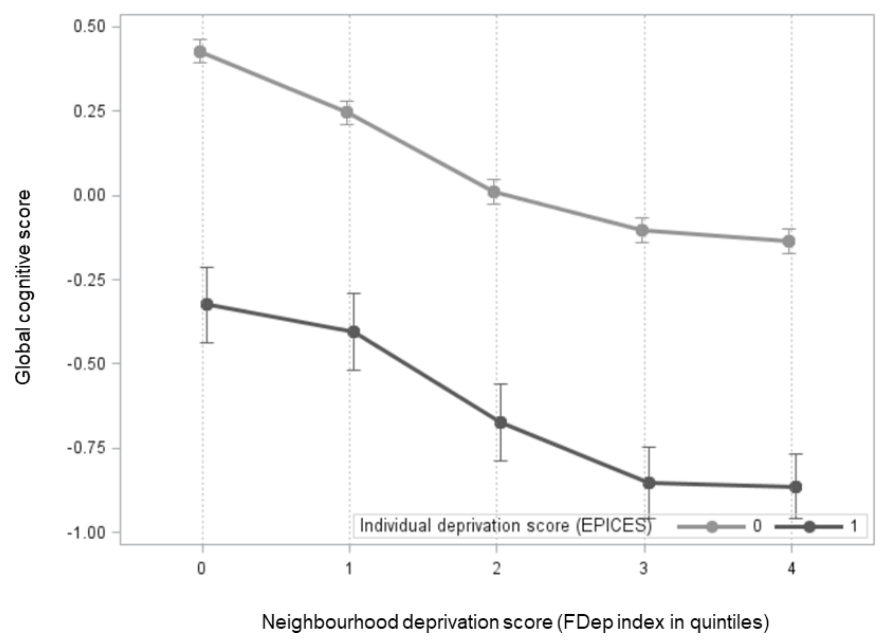

Figure 1 Distribution of the global cognitive score according to the neighbourhood deprivation level in the individually deprived and not deprived groups. FDep index, French Deprivation Index; EPICES, Evaluation of Deprivation and Inequalities in Health Screening Centres. deprivation (ref. Q1; Q2: OR=1.09, 95\% CI: 0.98 to 1.22; Q3: OR=1.15, 95\% CI: 1.04 to 1.27 ; $Q 4:$ OR $=1.15$, $95 \%$ CI: 1.04 to 1.28 ; Q5: OR=1.25, 95\% CI: 1.13 to 1.39 ).

The results of model 3 for each cognitive test are presented in supplementary data (online supplementary table 2). Only the DSST score was associated with the level of neighbourhood deprivation in model $3 \mathrm{~b}$ $(\mathrm{p}=0.005)$, with a socioeconomic gradient (ref. Q1; Q2: OR=1.03, 95\% CI: 0.94 to 1.13 ; Q3: OR=1.13, 95\% CI: 1.03 to 1.24; Q4: OR=1.15, 95\% CI: 1.04 to 1.26 ; Q5: $\mathrm{OR}=1.18$, $95 \%$ CI: 1.07 to 1.30 ). Moreover, the risk of lower scores at the TMT-A and VFT (phonemic fluency task) was higher for individuals living in the most deprived environment (respectively, ref. Q1; Q5: OR=1.12, 95\% CI: 1.02 to 1.22/ Q5: $\mathrm{OR}=1.10,95 \% \mathrm{CI}$ : 1.00 to 1.21 ).

Sensitivity analyses performed on a subsample with data on social isolation and physical activity $(n=11511)$ showed that the influence of neighbourhood deprivation remained significant after adjustment for these two factors (data not shown).

\section{Rural versus urban areas}

As urbanisation influenced the association between contextual deprivation and cognitive impairment ( $p$ of interaction $=0.01$ ), results were stratified according to the participants' rural or urban status (table 3). Among the 44648 participants, 7722 lived in rural areas (17\%). Living in a deprived neighbourhood was associated with cognitive impairment in both rural and urban dwellers (table 3). For rural dwellers, the association was significant only when they lived in a commune with the highest deprivation level (Q5 vs Q1: OR, 1.70; 95\% CI, 1.17 to 2.47). Conversely, this association was significant for urban dwellers who lived in a commune classified in quintiles 3,4 or 5 .

\section{DISCUSSION}

Our study shows that in a large sample $(n=44648)$ of middle-aged and young-old volunteers (45 to 69 years of age) from the French CONSTANCES cohort, cognitive impairment is influenced by individual and geographical deprivation, with a socioeconomic gradient. After adjustment for individual factors, the risk of cognitive impairment was higher for deprived individuals and individuals living in a deprived environment. The neighbourhood deprivation level was associated with the overall cognitive impairment (global cognitive score), and also with the impairment of specific cognitive skills, such as attention 
Table 2 Relationship between individual deprivation/neighbourhood deprivation and global cognitive score, based on multilevel logistic models $(n=44648)$

ORs $(95 \% \mathrm{Cl})$ per model

\begin{tabular}{|c|c|c|c|c|c|c|}
\hline \multirow[t]{2}{*}{ Global cognitive score } & \multicolumn{3}{|c|}{ Model 1} & \multicolumn{3}{|c|}{ Model 2} \\
\hline & OR & $95 \% \mathrm{Cl}$ & P value & OR & $95 \% \mathrm{Cl}$ & $P$ value \\
\hline \multicolumn{7}{|l|}{ Individual deprivation } \\
\hline Not deprived & Ref. & & $<0.0001$ & & & \\
\hline Deprived & 1.56 & $(1.46-1.67)$ & & & & \\
\hline \multicolumn{7}{|l|}{ FDep index } \\
\hline Q1 (least deprived) & & & & Ref. & - & $<0.0001$ \\
\hline Q2 & & & & 1.09 & $(0.98-1.20)$ & \\
\hline Q3 & & & & 1.15 & $(1.04-1.27)$ & \\
\hline Q4 & & & & 1.17 & $(1.05-1.29)$ & \\
\hline
\end{tabular}

Models 1 and 2 were adjusted for sex, age, education level, geographical origin, HSC, smoking, alcohol consumption, BMI, HBP, diabetes, cardiovascular diseases and depressive symptomatology.

BMI, body mass index; EPICES, Evaluation of Deprivation and Inequalities in Health Screening Centres; FDep index, French Deprivation Index; HBP, high blood pressure; HSC, health screening centre.

and executive functions, independently of the individual deprivation level. This association concerned mainly the VFT (phonemic fluency task) and the DSST that has the best psychometric proprieties, ${ }^{27}$ and may be first affected during the long-term pre-dementia process. ${ }^{28}$ Moreover, low psychomotor speed could be a marker of brain vulnerability. ${ }^{29}$

Our findings are in accordance with those reported by our team on the dementia risk in the Three City Study cohort (a cohort of $\geq 65$-year-old participants), ${ }^{17} 18$

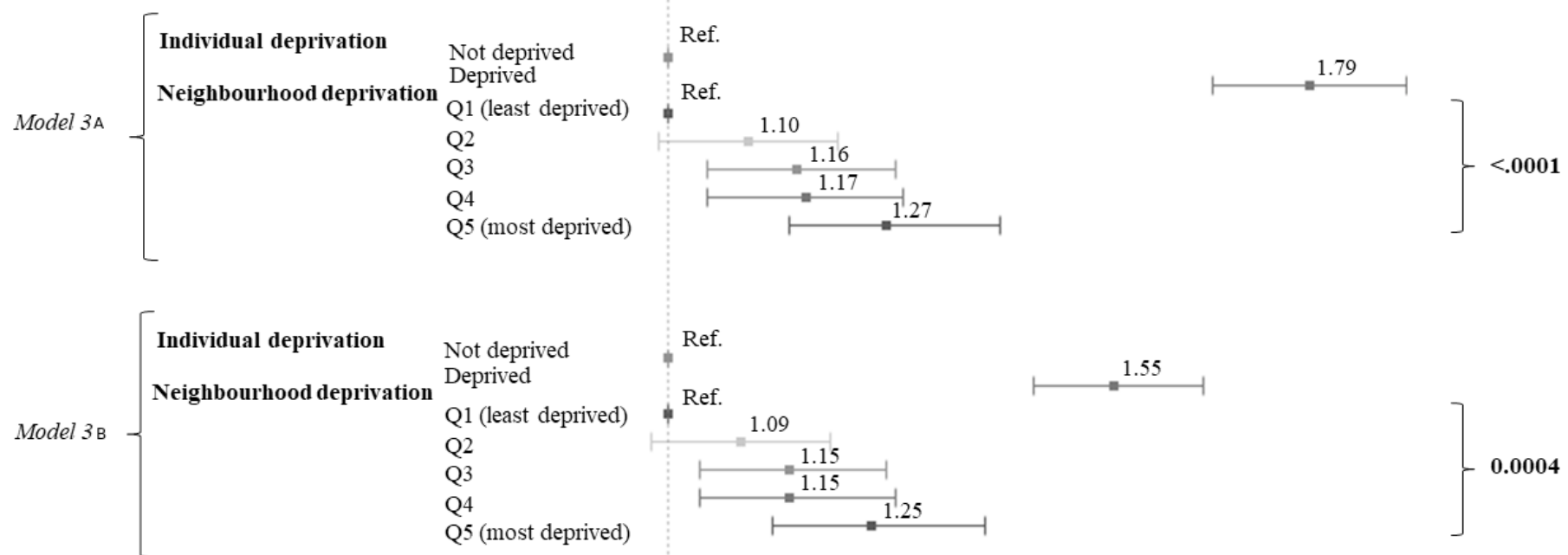

\begin{tabular}{lllllllllll}
0.9 & 1.0 & 1.1 & 1.2 & 1.3 & 1.4 & 1.5 & 1.6 & 1.7 & 1.8 & 1.9 \\
& \multicolumn{1}{c}{ Odds Ratio $(95 \% \mathrm{CI})$}
\end{tabular}

Figure 2 Association between individual deprivation/neighbourhood deprivation and global cognitive impairment, based on multilevel logistic models $(n=44648)$. Model 3: individual deprivation and neighbourhood deprivation were considered in the same model. Model 3A: adjusted for sex, age, education level and HSC. Model 3B: adjusted like for model 3A and also for geographical origin, smoking, alcohol consumption, BMI, HBP, diabetes, cardiovascular diseases and depressive symptomatology. BMI, body mass index; HBP, high blood pressure; HSC, health screening centre. 


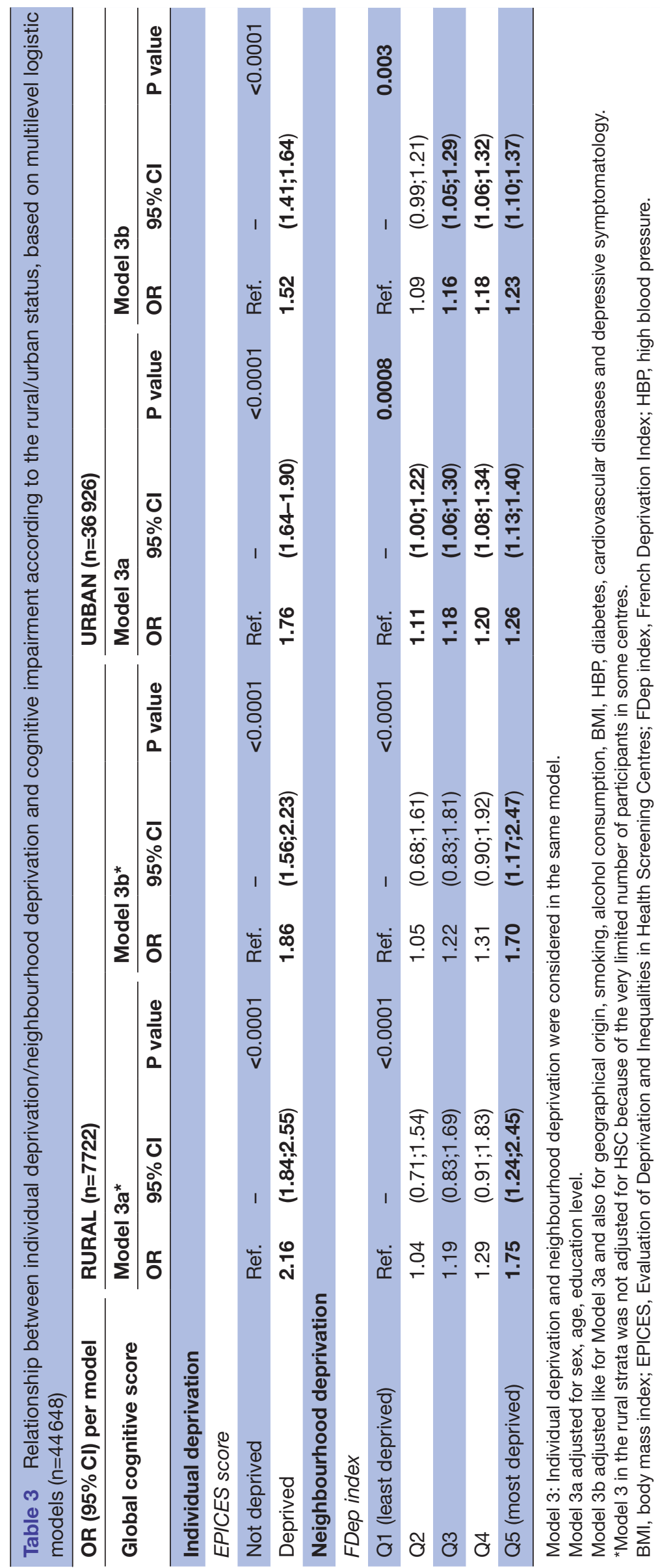


although our current work concerned a middle-aged and young-old population. The present study shows that the impact of environmental deprivation on cognition might be detected earlier in life. To our knowledge, only one study showed an interaction between neighbourhood psychosocial hazards and presence of the APOE $\varepsilon 4$ allele $^{30}$ which is the main genetic determinant of Alzheimer's disease risk, in a group of urban dwellers aged $50-70$ years.

The environmental deprivation level could be associated with qualitative and quantitative differences in resources (eg, sports equipment/recreation centres; cultural equipment: museums, libraries, bookstores, cinema; healthy food stores; public transport; and health services), social environment (social stressors; social cohesion, community centres, social clubs) and built environment (presence of parks/green spaces, street connectivity). ${ }^{11} 2031$ The lower availability of these resources could affect individual competences through a lower active lifestyle (eg, physical activity), lower social activity and support, limited healthy lifestyles or higher level of anxiety and depression. All these factors may increase the risk of cognitive impairment. The socioeconomic environment could also influence cardiovascular risk factors and morbidity (myocardial infarction, stroke, coronary heart disease and CVD mortality ${ }^{32}$ ) that are associated with higher risk of dementia. ${ }^{33}$ On the other hand, the environment can also act as a source of 'brain training', by offering cognitive stimulation. ${ }^{34}$ Cognitive reserve is built through relevant everyday experiences, including leisure and social activities, and more generally through living conditions that offer mental stimulation and that can be influenced directly or indirectly by the living environment characteristics. $^{20} 35$

Multiple environmental influences can contribute to urban/rural differences. The relatively few studies on subjects living in rural areas showed higher prevalence of cognitive impairment and dementia. ${ }^{36}{ }^{37}$ Urban areas could provide a more exciting and intellectually demanding (eg, higher level of visually and auditory stimuli) environment than rural areas. ${ }^{20}$ However, urban areas may provide 'toxic social circumstances' (fragmentation of family structure and social support, wider socioeconomic disparities, reduced social cooperation) that facilitate chronic stress and abnormal neural development in vulnerable individuals. ${ }^{19}$

One of the main strengths of this study is the large population sample from the general population in metropolitan France. CONSTANCES is a large-sized cohort that includes randomly selected participants who live in urban and rural neighbourhoods with variable socioeconomic status. Unlike many other studies, the effect of deprivation on cognitive performance was examined in middle-aged and young-old participants (45 to 70 years of age). In the CONSTANCES cohort, a cognitive test battery was administered by neuropsychologists in standardised conditions. In addition, deprivation was evaluated with validated tools that are commonly used in many French studies on deprivation. ${ }^{6}{ }^{12} 24$ The EPICES score allowed taking into account the multidimensional nature of precariousness, by including markers of individual resources and also of social support. To reduce the possibility of residual confounding, analyses were controlled for many individual variables (socio-demographic characteristics, lifestyle and health status) and were performed using multilevel models adapted to the specific structure of the data.

Limitations of this study include the cross-sectional analysis of cognitive performance data. Due to the study observational design, the possibility of residual confounding cannot be ruled out. The absence of data on APOEE4 carrier status did not allow examining its potential modifying effect. The neighbourhood deprivation level of the place of residence was evaluated at inclusion, without considering each participant's residential history and the time spent outside the area of residence each day, for instance at work. The limits of the area of influence are difficult to assess and generally administrative boundaries are used to define the neighbourhood environment. ${ }^{10}$ Furthermore, it would be interesting to include other environmental dimensions that can influence cognitive decline (eg, pollution (more frequent in urban areas), presence of green spaces, noise and environmental layout) and that might help to better interpret our results. ${ }^{38-40}$

This study in a large sample of the middle-aged and young-old French population provides new evidence that the socio-economic level of the place of residence affects the cognitive performance of people in this age group. Higher neighbourhood deprivation was associated with worse cognition after controlling for individual deprivation and many individual factors. These results stress the need of preventive strategies to target socially deprived at-risk populations and at-risk geographical areas.

\section{Author affiliations}

${ }^{1}$ Neuropsychiat Epidemiol \& Clin Res, Univ Montpellier, INSERM, Montpellier, France ${ }^{2}$ IRD - Hydrosciences UMR 5569, Montpellier University, Montpellier, France ${ }^{3}$ Harvard Center for Population and Development Studies, Harvard University, Cambridge, Massachusetts, USA

${ }^{4}$ Paris Descartes University, Paris, France

${ }^{5}$ INSERM, Population-Based Epidemiological Cohorts Research Unit, UMS 011, Villejuif, France

${ }^{6}$ UVSQ, INSERM, VIMA; Aging and chronic diseases, U1168, Villejuif, France ${ }^{7}$ Memory Research and Resources Center, Department of Neurology, Montpellier University Hospital Gui de Chauliac, Montpellier, France

Contributors NL performed the statistical analysis, interpreted the results and edited the first draft of the manuscript. IC and EC also contributed to the statistical analysis. LB was involved in the conceptualisation and interpretation of deprivation scores. MG and $\mathrm{MZ}$ acquired the data. $\mathrm{CB}$ designed the study, contributed data and helped to write the manuscript. NL and CB developed the study hypotheses. All authors critically revised the manuscript.

Funding The CONSTANCES Cohort Study was supported and funded by the 'Caisse nationale d'assurance maladie' (CNAM). The CONSTANCES Cohort Study is an 'Infrastructure nationale en Biologie et Santé' and benefits from a grant from Agence Nationale de la Recherche (ANR-11-INBS-0002). CONSTANCES is also partly funded by MSD, AstraZeneca and Lundbeck. The present analyses were supported by a grant from the French Agency for Food, Environmental and Occupational Health \& Safety (ANSES, EST - №2016/1/102).

Competing interests None declared. 
Patient and public involvement Patients and/or the public were not involved in the design, or conduct, or reporting, or dissemination plans of this research.

Patient consent for publication Not required.

Ethics approval The Constances cohort received ethical approval from the French National Data Protection Authority (authorisation no.910486) and the Institutional Review Board of the National Institute for Medical Research (authorisation no.01011). All participants gave informed consent to participate.

Provenance and peer review Not commissioned; externally peer reviewed.

Data availability statement Data are available upon reasonable request. The Constances cohort is available for registered users and on approval of a research project by the scientific board at http://www.constances.fr/conduct-projectongoing.php.

Open access This is an open access article distributed in accordance with the Creative Commons Attribution Non Commercial (CC BY-NC 4.0) license, which permits others to distribute, remix, adapt, build upon this work non-commercially, and license their derivative works on different terms, provided the original work is properly cited, appropriate credit is given, any changes made indicated, and the use is non-commercial. See: http://creativecommons.org/licenses/by-nc/4.0/.

\section{ORCID iD}

Noémie Letellier http://orcid.org/0000-0002-6925-1217

\section{REFERENCES}

1 Townsend P. Deprivation. J Soc Policy 1987;16:125-46.

2 Braveman P, Gottlieb L. The social determinants of health: it's time to consider the causes of the causes. Public Health Rep 2014;129 Suppl 2:19-31.

3 Marmot M. The influence of income on health: views of an epidemiologist. Health Aff 2002;21:31-46.

4 Artazcoz L, Rueda S. Social inequalities in health among the elderly: a challenge for public health research. J Epidemiol Community Health 2007;61:466-7.

5 Bongue B, Colvez A, Amsallem E, et al. Assessment of health inequalities among older people using the EPICES score: a composite index of social deprivation. J Frailty Aging 2016;5:168-73.

6 Labbe E, Blanquet M, Gerbaud L, et al. A new reliable index to measure individual deprivation: the EPICES score. Eur J Public Health 2015;25:604-9.

7 Yaffe K, Falvey C, Harris TB, et al. Effect of socioeconomic disparities on incidence of dementia among biracial older adults: prospective study. BMJ 2013;347:f7051.

8 Zeki Al Hazzouri A, Haan MN, Kalbfleisch JD, et al. Life-Course socioeconomic position and incidence of dementia and cognitive impairment without dementia in older Mexican Americans: results from the Sacramento area Latino study on aging. Am J Epidemiol 2011;173:1148-58.

9 Bosma H, van de Mheen HD, Borsboom GJ, et al. Neighborhood socioeconomic status and all-cause mortality. Am J Epidemiol 2001;153:363-71.

10 Besser LM, McDonald NC, Song Y, et al. Neighborhood environment and cognition in older adults: a systematic review. Am J Prev Med 2017;53:241-51.

11 Rosso AL, Flatt JD, Carlson MC, et al. Neighborhood socioeconomic status and cognitive function in late life. Am J Epidemiol 2016;183:1088-97.

12 Ouvrard C, Meillon C, Dartigues J-F, et al. Do individual and geographical deprivation have the same impact on the risk of dementia? A 25-year follow-up study. J Gerontol Ser B 2017;36.

13 Lang IA, Llewellyn DJ, Langa KM, et al. Neighborhood deprivation, individual socioeconomic status, and cognitive function in older people: analyses from the English longitudinal study of ageing. J Am Geriatr Soc 2008;56:191-8.

14 Sheffield KM, Peek MK. Neighborhood context and cognitive decline in older Mexican Americans: results from the Hispanic established populations for epidemiologic studies of the elderly. Am J Epidemiol 2009;169:1092-101.

15 Clarke PJ, Weuve J, Barnes L, et al. Cognitive decline and the neighborhood environment. Ann Epidemiol 2015;25:849-54.

16 Cadar D, Lassale C, Davies $\mathrm{H}$, et al. Individual and area-based socioeconomic factors associated with dementia incidence in England: evidence from a 12-year follow-up in the English longitudinal study of ageing. JAMA Psychiatry 2018;75:723-32.

17 Letellier N, Carrière I, Gutierrez L-A, et al. Influence of activity space on the association between neighborhood characteristics and dementia risk: results from the 3-City study cohort. BMC Geriatr 2019;19:4.

18 Letellier N, Gutierrez L-A, Carrière I, et al. Sex-Specific association between neighborhood characteristics and dementia: the Three-City cohort. Alzheimers Dement 2018;14:473-82.

19 Tost H, Champagne FA, Meyer-Lindenberg A. Environmental influence in the brain, human welfare and mental health. Nat Neurosci 2015;18:1421-31.

20 Cassarino M, Setti A. Environment as 'Brain Training': A review of geographical and physical environmental influences on cognitive ageing. Ageing Res Rev 2015;23:167-82.

21 Zins M, Goldberg M, team C, CONSTANCES team. The French CONSTANCES population-based cohort: design, inclusion and follow-up. Eur J Epidemiol 2015;30:1317-28.

22 Abrantes P, Sabatier S, Guenot C. Le score EPICES : I'indicateur de précarité des Centres d'examens de santé de l'Assurance Maladie. 18, 2005.

23 Sass C, Guéguen R, Moulin JJ, et al. Comparison of the individual deprivation index of the French Health Examination Centres and the administrative definition of deprivation]. Sante Publique VandoeuvreNancy Fr 2006;18:513-22.

24 Rey G, Jougla E, Fouillet A, et al. Ecological association between a deprivation index and mortality in France over the period 1997 . 2001: variations with spatial scale, degree of urbanicity, age, gender and cause of death. BMC Public Health 2009;9:33.

25 Ouvrard C, Berr C, Meillon C, et al. Norms for standard neuropsychological tests from the French CONSTANCES cohort. Eur J Neurol 2019;26:786-93.

26 Austin PC, Merlo J. Intermediate and advanced topics in multilevel logistic regression analysis. Stat Med 2017;36:3257-77.

27 Mura T, Amieva H, Goldberg M, et al. Effect size for the main cognitive function determinants in a large cross-sectional study. Eur J Neurol 2016;23:1614-26.

28 Amieva H, Mokri H, Le Goff M, et al. Compensatory mechanisms in higher-educated subjects with Alzheimer's disease: a study of 20 years of cognitive decline. Brain 2014;137:1167-75.

29 Amieva $\mathrm{H}$, Meillon C, Proust-Lima C, et al. Is low psychomotor speed a marker of brain vulnerability in late life? digit symbol substitution test in the prediction of Alzheimer, Parkinson, stroke, disability, and depression. Dement Geriatr Cogn Disord 2019;47:297-305.

30 Lee BK, Glass TA, James BD, et al. Neighborhood psychosocial environment, apolipoprotein $\mathrm{E}$ genotype, and cognitive function in older adults. Arch Gen Psychiatry 2011;68:314-21.

31 Wu Y-T, Prina AM, Brayne C. The association between community environment and cognitive function: a systematic review. Soc Psychiatry Psychiatr Epidemiol 2015;50:351-62.

32 Havranek EP, Mujahid MS, Barr DA, et al. Social determinants of risk and outcomes for cardiovascular disease. Circulation 2015;132:873-98.

33 Gottesman RF, Albert MS, Alonso A, et al. Associations between midlife vascular risk factors and 25-year incident dementia in the Atherosclerosis risk in communities (ARIC) cohort. JAMA Neurol 2017;74:1246-54.

34 Clarke PJ, Ailshire JA, House JS, et al. Cognitive function in the community setting: the neighbourhood as a source of 'cognitive reserve'? J Epidemiol Community Health 2012;66:730-6.

35 Cassarino M, Setti A. Complexity as key to designing CognitiveFriendly environments for older people. Front Psychol 2016;7:1329.

36 Russ TC, Batty GD, Hearnshaw GF, et al. Geographical variation in dementia: systematic review with meta-analysis. Int J Epidemiol 2012;41:1012-32.

37 Contador I, Bermejo-Pareja F, Puertas-Martin V, et al. Childhood and adulthood rural residence increases the risk of dementia: NEDICES study. Curr Alzheimer Res 2015;12:350-7.

38 Killin LOJ, Starr JM, Shiue IJ, et al. Environmental risk factors for dementia: a systematic review. BMC Geriatr 2016;16:175.

39 de Keijzer C, Tonne C, Basagaña X, et al. Residential surrounding Greenness and cognitive decline: a 10-year follow-up of the Whitehall II cohort. Environ Health Perspect 2018;126:077003.

40 Ailshire J, Karraker A, Clarke P. Neighborhood social stressors, fine particulate matter air pollution, and cognitive function among older U.S. adults. Soc Sci Med 2017;172:56-63. 